\title{
Micromotors for active delivery of minerals toward the treatment of iron deficiency anemia
}

\author{
Emil Karshalev $\ddagger$, Yue Zhang $\neq$, Berta Esteban-Fernández de Ávilał, Mara Beltrán- \\ Gastélum, Yijie Chen, Rodolfo Mundaca-Uribe, Fangyu Zhang, Bryan Nguyen, Yao
}

Tong, Ronnie H. Fang, Liangfang Zhang ${ }^{*}$ and Joseph Wang*

Department of NanoEngineering, Chemical Engineering Program, University of

California San Diego, La Jolla, CA 92093, USA.

‡These authors contributed equally to this work

Corresponding Author

*Tel: 858-246-0128; email: josephwang@eng.ucsd.edu (J. W.)

*Tel: 858-246-0999; email: zhang@ucsd.edu (L.Z.)

\section{Supporting Information}

Figures S1-S3

Tables S1 and S2

Video S1 Legend 


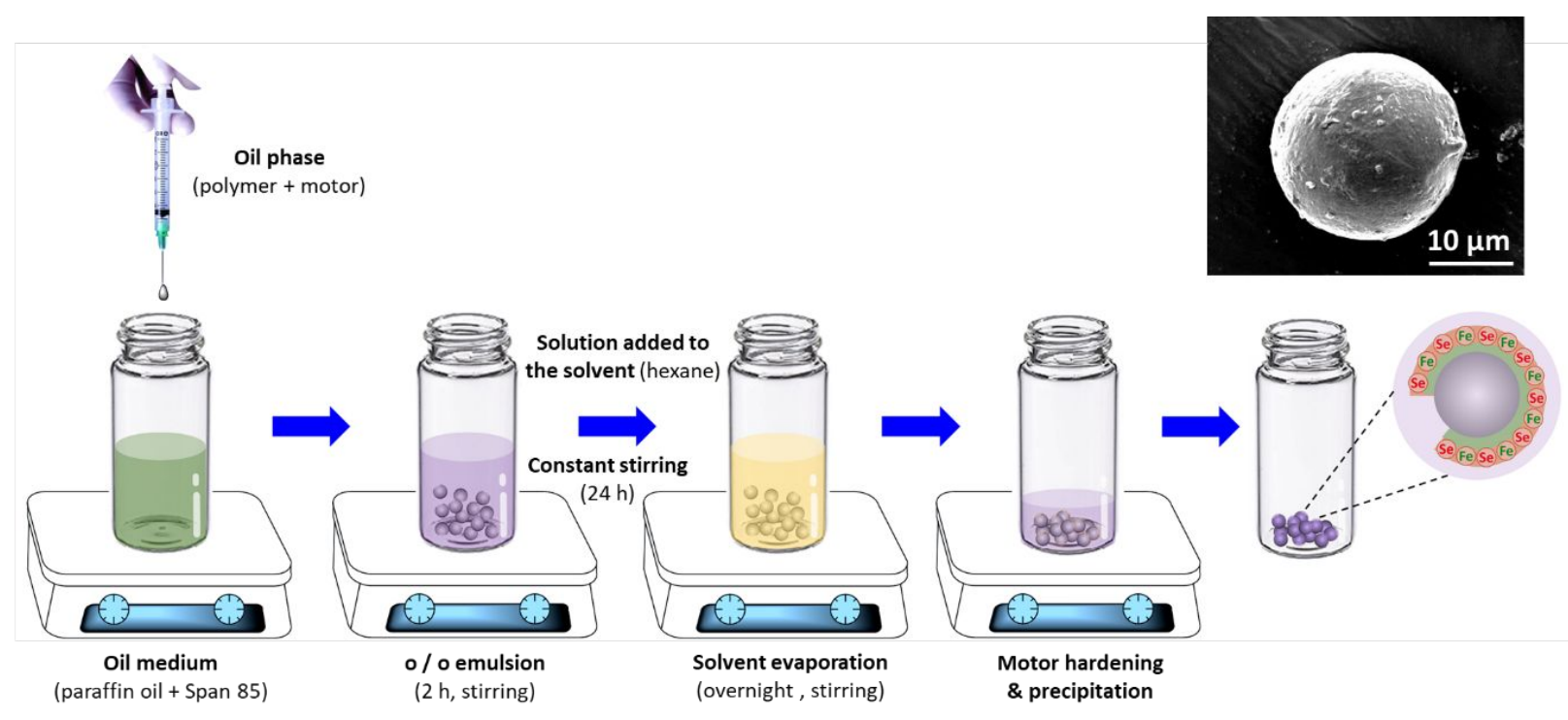

Figure S1. Enteric layer coating process. Firstly, enteric polymer Eudragit L100-55 in isopropyl alcohol and micromotors are added to paraffin oil/Span 85 mixture. The mixture is stirred for $2 \mathrm{~h}$ and then hexane is added followed by $24 \mathrm{~h}$ stirring. Finally, the hexane and alcohol solvents are evaporated followed by a mild annealing step to harden the enteric shell. SEM image of an $\mathrm{Fe} / \mathrm{Se}$-loaded micromotor with enteric coating is shown as an inset.

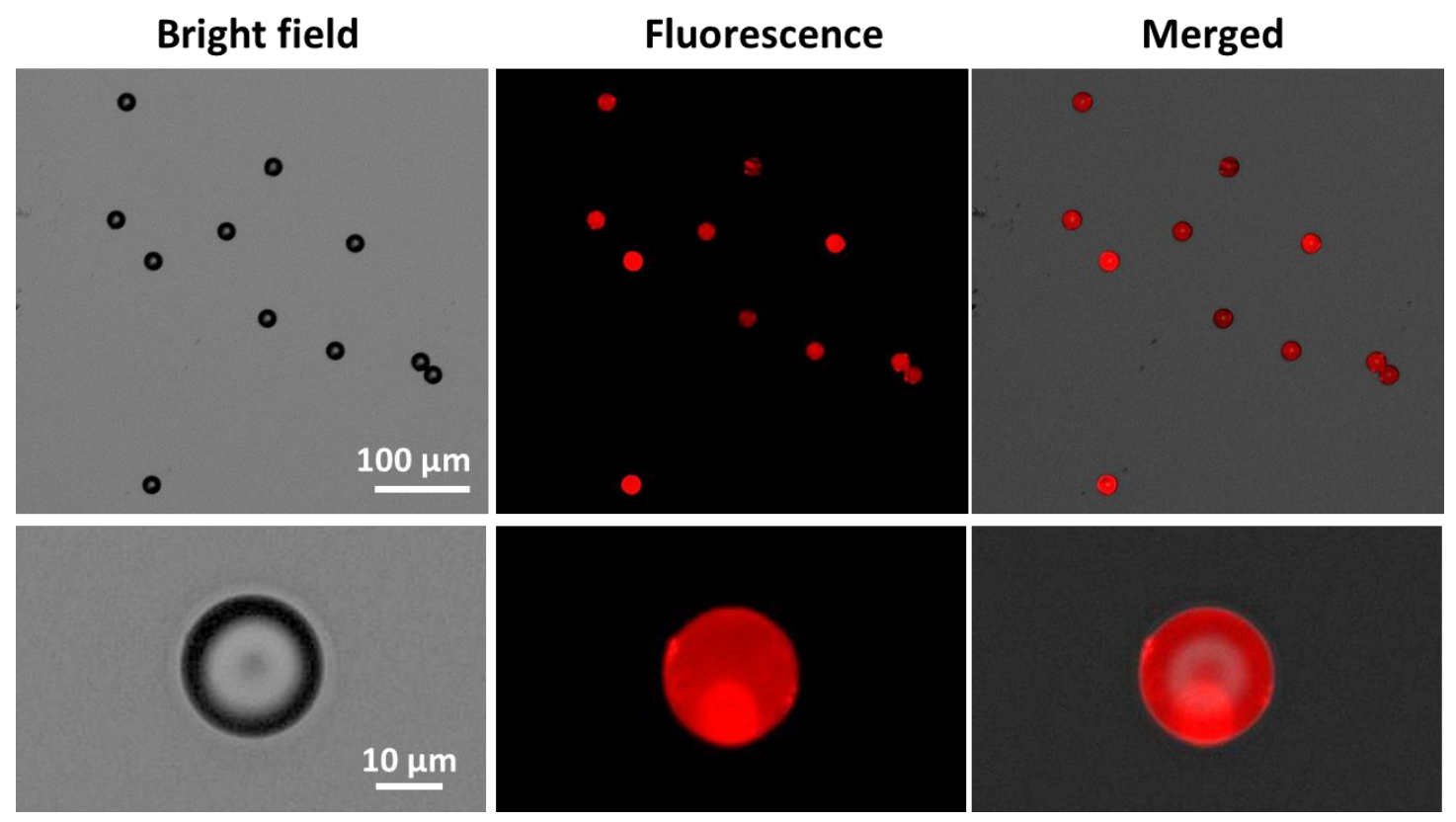

Figure S2. Brightfield, fluorescence, and merged images of inert silica particles loaded with minerals analogously to $\mathrm{Mg}$-based micromotors and used as negative controls. Fluorescent dye used is Rhodamine-6G (Rh-6G, $\left.\lambda_{\text {ex }}=524 \mathrm{~nm} / \lambda_{\text {em }}=547 \mathrm{~nm}\right)$. 


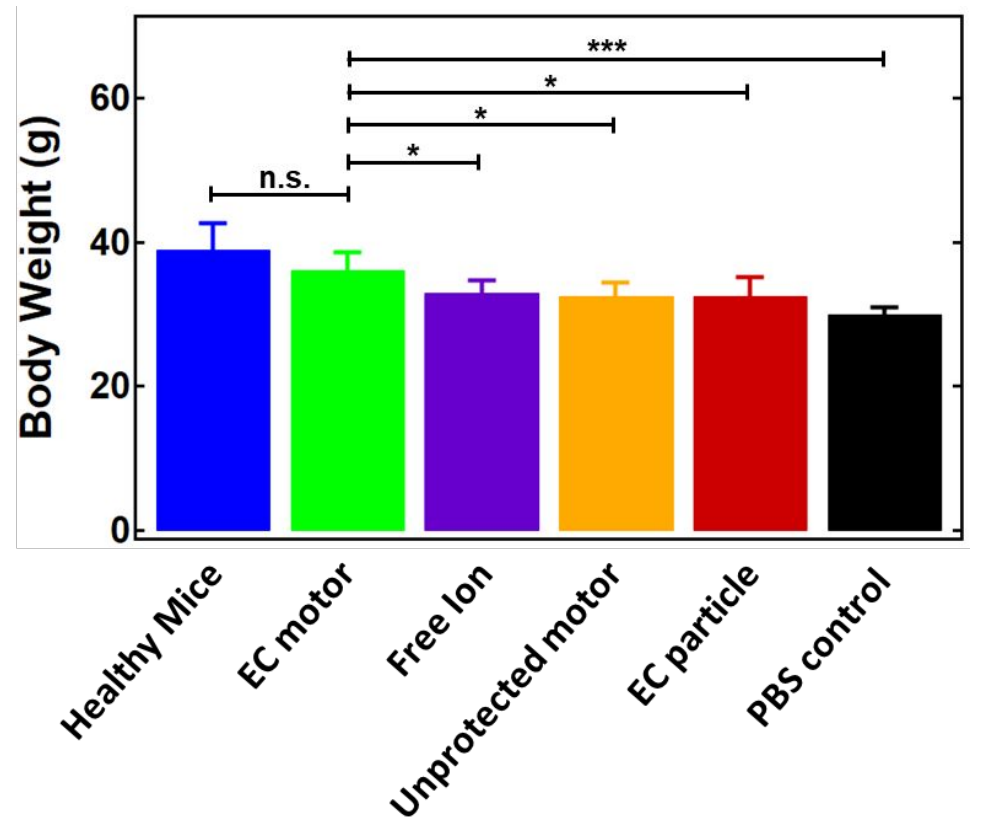

Figure S3. Weight of mice after 30 days of treatment.

Table S1. Calculation of daily recommended values and upper limits of minerals for mice. Recommended daily values for iron $(\mathrm{Fe})$, selenium $(\mathrm{Se})$, and magnesium $(\mathrm{Mg})$ were obtained from Ref. 1 while upper daily limits were obtained from Ref. 2 for humans. These values were converted to dosages for mice by using a mass ratio of average mouse weight $(35 \mathrm{~g})$ divided by average human weight $\left(70 \mathrm{~kg}\right.$ ) and multiplication by the correction factor, $\mathrm{K}_{\mathrm{m}}(12.3$ for mice). Correction factor was obtained from Ref. 3 .

\begin{tabular}{|c|c|c|c|c|c|c|c|c|c|}
\hline & \multicolumn{3}{|c|}{ Iron (Fe) } & \multicolumn{3}{|c|}{ Selenium (Se) } & \multicolumn{3}{|c|}{ Magnesium (Mg) } \\
\hline \multirow{2}{*}{$\begin{array}{l}\text { Recommended } \\
\text { Human Dosage }\end{array}$} & $\begin{array}{l}\text { Daily } \\
\text { Min } \\
\text { (mg/d) }\end{array}$ & $\begin{array}{l}\text { Daily } \\
\text { Max } \\
(\mathrm{mg} / \mathrm{d})\end{array}$ & $\begin{array}{l}\text { Upper Daily } \\
\text { Limit (mg/d) }\end{array}$ & $\begin{array}{l}\text { Daily } \\
\text { Min } \\
(\mu \mathrm{g} / \mathrm{d})\end{array}$ & $\begin{array}{l}\text { Daily } \\
\text { Max } \\
(\mu \mathrm{g} / \mathrm{d})\end{array}$ & $\begin{array}{l}\text { Upper Daily } \\
\text { Limit }(\mu \mathrm{g} / \mathrm{d})\end{array}$ & $\begin{array}{l}\text { Daily } \\
\text { Min } \\
(\mathrm{mg} / \mathrm{d})\end{array}$ & $\begin{array}{l}\text { Daily } \\
\text { Max } \\
(\mathrm{mg} / \mathrm{d})\end{array}$ & $\begin{array}{l}\text { Upper Daily } \\
\text { Limit }(\mathrm{mg} / \mathrm{d})^{\mathrm{a}}\end{array}$ \\
\hline & 8 & 15 & 45 & 40 & 55 & 400 & 240 & 420 & 350 \\
\hline \multirow{2}{*}{$\begin{array}{l}\text { Recommended } \\
\text { Mouse Dosage }\end{array}$} & $\begin{array}{l}\text { Daily } \\
\text { Min } \\
(\mu \mathrm{g} / \mathrm{d})\end{array}$ & $\begin{array}{l}\text { Daily } \\
\text { Max } \\
(\mu \mathrm{g} / \mathrm{d})\end{array}$ & $\begin{array}{l}\text { Upper Daily } \\
\text { Limit }(\mu \mathrm{g} / \mathrm{d})\end{array}$ & $\begin{array}{l}\text { Daily } \\
\text { Min } \\
\text { (ng/d) }\end{array}$ & $\begin{array}{l}\text { Daily } \\
\text { Max } \\
\text { (ng/d) }\end{array}$ & $\begin{array}{l}\text { Upper Daily } \\
\text { Limit (ng/d) }\end{array}$ & $\begin{array}{l}\text { Daily } \\
\text { Min } \\
(\mu \mathrm{g} / \mathrm{d})\end{array}$ & $\begin{array}{l}\text { Daily } \\
\text { Max } \\
(\mu \mathrm{g} / \mathrm{d})\end{array}$ & $\begin{array}{l}\text { Upper Daily } \\
\text { Limit }(\mu \mathrm{g} / \mathrm{d})\end{array}$ \\
\hline & 49 & 92 & 277 & 246 & 338 & 2460 & 1476 & 2583 & 2153 \\
\hline
\end{tabular}

a. Value given is only for $\mathrm{Mg}$ supplements and does not represent overall $\mathrm{Mg}$ daily intake. 
Table S2. Description of the structural and chemical composition of the Fe/Se-loaded micromotors and other control groups (free ion, unprotected micromotor, passive particle and PBS).

\begin{tabular}{|c|c|c|c|c|c|}
\hline & Core & Conformal layer & Protective layer & Mineral carrier* & Enteric coating \\
\hline $\begin{array}{c}\text { Fe/Se-loaded } \\
\text { micromotor }\end{array}$ & $\begin{array}{c}\mathrm{Mg} \\
\text { microparticle }\end{array}$ & $\mathrm{TiO}_{2}$ & PLGA & $\begin{array}{c}\mathrm{Fe} / \mathrm{Se}-\mathrm{loaded} \\
\text { chitosan }\end{array}$ & Eudragit $^{\circledR}$ L100-55 \\
\hline Free ion ${ }^{\dagger}$ & - & - & - & Free $\mathrm{Fe} / \mathrm{Se}$ & - \\
\hline $\begin{array}{l}\text { Unprotected } \\
\text { micromotor }\end{array}$ & $\begin{array}{c}\mathrm{Mg} \\
\text { microparticle }\end{array}$ & $\mathrm{TiO}_{2}$ & PLGA & $\begin{array}{l}\text { Fe/Se-loaded } \\
\text { chitosan }\end{array}$ & - \\
\hline $\begin{array}{l}\text { Passive } \\
\text { particle }\end{array}$ & $\begin{array}{c}\text { Silica } \\
\text { microparticle }\end{array}$ & - & - & $\begin{array}{c}\text { Fe/Se-loaded } \\
\text { chitosan }\end{array}$ & Eudragit ${ }^{\circledR}$ L100-55 \\
\hline PBS & - & - & - & - & - \\
\hline
\end{tabular}

*Fe and Se were present at the same level in all the control formulations.

${ }^{\dagger}$ Free ion formulations are the aqueous extracts of equivalent amounts of $\mathrm{Fe} / \mathrm{Se}$-loaded micromotors in dilute $\mathrm{HCl}$

Video S1. Propulsion of non-loaded and mineral-loaded micromotors in simulated gastric fluid.

\section{References}

(1) In DRI Dietary Reference Intakes Calcium and Vitamin D; Ross, A. C., Taylor, C. L., Yaktine, A. L., Del Valle, H. B., Eds. The National Academies Press: Washington, D. C., 2011; pp1108-1109.

(2) In DRI Dietary Reference Intakes Calcium and Vitamin D; Ross, A. C., Taylor, C. L., Yaktine, A. L., Del Valle, H. B., Eds. The National Academies Press, Washington, D. C., 2011; pp 1114-1115.

(3) Nair, A. B., Jacob, S., A simple practice guide for dose conversion between animals and human. J. Basic Clin. Pharma. 2016, 7, 27-31. 